\title{
Research Paper \\ The Relationship Between Functional Movement Screening With Fitness Factors in Female Athlete Students
}

\author{
Maral Entezami $^{* 1}\left(\mathbb{C}\right.$, Hasan Daneshmandi $^{1}(\mathbb{C})$, Ali Shamsi Majelan ${ }^{1}$ (1)
}

1. Department of Sport Injuries and Corrective Exercises, Faculty of Physical Education and Sports Sciences, University of Guilan, Rasht, Iran.

$\begin{aligned} & \text { Use yourdevice to scan } \\ & \text { and read the article online }\end{aligned}$
With Fitness Factors in Female Athlete Students (Persian)]. Journal of Sport Biomechanics. 2021; 6(4):264-275. https://doi.
org/10.32598/biomechanics.6.3.5
doishttps://doi.org/10.32598/biomechanics.6.3.5

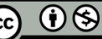

Article Info:

Received: 12 May 2019

Accepted: 14 Oct 2019

Available Online: 01 Mar 2021

Keywords:

Functional Movement Screening test, Athletes, Fitness

\section{A B STRACT}

Objective The study examines the relationship between Functional Movement Screening (FMS) tests with fitness factors in female athlete students.

Methods The study population includes 30 female athlete students (Mean \pm SD of age: 23.36 \pm 3.10 ; height: 163.45 \pm 5.06 ; and 57.40 \pm 6.43 ). FMS test was used for measuring movement patterns. Sargent test was used to measure lower limb power, Y-test was used to measure dynamic balance, Stork test was used to measure static balance, and flexor/extensor muscle endurance tests were also used to measure trunk muscle endurance. In addition, Pearson correlation was used to evaluate the relationship between scores of functional motion screening and fitness factors. Multivariate regression was used to solve the linear regression equation. The data analyzed in SPSS software, V. 22 at significance level of $P \geq 0.05$.

Results Our results showed no significant relationship between FMS test with fitness factors $(P>0.05)$.

Conclusion There is no significant relationship between total score of FMS test with fitness factors. It seems that FMS addresses the quality of basic motor activities while fitness factors determine sport skills; thus, FMS scores can't explain fitness factors.

\section{Extended Abstract}

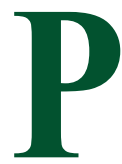

\section{Introduction}

articipation in sports activities is increasing, and in addition to its potential benefits, it undoubtedly brings the possibility of injury and negative consequences such as neuromuscular injuries for each person [1]. $10.19 \%$ of all acute injuries treated in hospitals are due to participation in sports activities. These sports injuries may impair a person's athletic performance in later life and limit a person's ability to perform painless movements, and even these sports injuries can cause the athlete to say goodbye to his sporting profession [2] permanently. Therefore, experts seek screening tests that identify people who are prone to injury to minimize the incidence of sports injuries while participating in sports activities [3, 4]; Therefore, to reduce these sports injuries, tools that have a high accuracy of diagnosis in terms of susceptibility to injury should be used.

The reason why the relationship between Functional Movement Screening (FMS) test scores and physical fitness factors differs, as Moore et al. Showed in their study, can be that when concluding about predicting FMS test injury, many factors must be considered and detailed. Therefore, this study aims to study the relationship between the FMS test and some physical fitness factors in girls who ex-

\section{* Corresponding Author:}

Maral Entezami, PhD.

Address: Department of Sport Injuries and Corrective Exercises, Faculty of Physical Education and Sports Sciences, University of Guilan, Rasht, Iran.

Tel: +98 (991) 6165992

E-mail: entezamidorsa2018@gmail.com 
ercise regularly to determine whether screening test scores that examine movement patterns and basal movements are significantly correlated with fitness factors that challenge motor and athletic skills?

\section{Methode}

This study is correlational, and the statistical population of it consisted of university students in the age group of 18 to 30 years, and according to the inclusion and exclusion criteria of the study, 30 people were randomly selected in the field of physical education. Subjects with no history of injury in the past year and no damage to the visual system, atrium, and the absence of neurological diseases and postural abnormalities (such as lordosis, flat feet, knee braces or cruciate ligament, etc.) affected the research process. All subjects agreed to participate in this study. According to the sources, athletes exercised at least three sessions a week, one to one and a half hours per session [17].

The FMS (ICC $=0.89)$ tests of Cook et al. were used in functional screening research [18]. In this study, descriptive statistics were used to describe each group's data (age, height, weight, and body mass index) and describe the data, and the Mean \pm SD was used. Shaper Wilk test was used to check the normality of the data. Since the data were averaged, the Pearson correlation coefficient was used to examine the relationship between the FMS test scores and fitness factors and multivariate regression to determine the linear regression equation. All data were collected in SPSS V. 22 , and the significance level in this study was considered $\mathrm{P} \geq 0.05$.

\section{Results}

Table 1 shows the Pearson correlation test results between the functional screening test's total score with some fitness factors. According to these results, there is no significant relationship between the functional screening test scores and the measured fitness factors.

\section{Discussion and Conclusion}

This study aimed to investigate the relationship between the FMS test and some fitness factors of female student-athletes. The results of the study showed that the measured fitness factors did not have a significant relationship with the FMS test's total score. We discuss the relationship between each of the measured fitness factors and the total FMS test score.

However, this study had some limitations, including not examining the relationship between each item of the FMS test and any fitness tests to evaluate the study results more accurately. Also, do not examine whether gender is involved in the relationship between fitness factors and FMS score. Therefore, it is suggested each item of the FMS test

Table 1. Mean $\pm \mathrm{SD}$ of measured variables and Pearson correlation test results between the total score of the functional screening test with some fitness factors of the subjects

\begin{tabular}{|c|c|c|c|c|}
\hline \multirow{2}{*}{ Fitness Factors } & \multirow{2}{*}{ Mean \pm SD } & \multicolumn{3}{|c|}{ Total Score of FMS Test } \\
\hline & & $\mathbf{R}$ & $r^{2}$ & Sig. \\
\hline Explosive power of the foot $(\mathrm{cm})$ & $34.40 \pm 5.53$ & 0.06 & 0.00 & 0.752 \\
\hline Static balance (seconds) & $12.68 \pm 5.70$ & 0.017 & 0.00 & 0.928 \\
\hline Dynamic balance (percentage of leg length) & $83.98 \pm 8.44$ & 0.062 & 0.00 & 0.743 \\
\hline Trunk flexor muscle endurance (sec) & $109.89 \pm 58.49$ & 0.019 & 0.00 & 0.858 \\
\hline Trunk extensor muscle endurance (s) & $107.99 \pm 28.66$ & 0.135 & 0.01 & 0.478 \\
\hline
\end{tabular}

Table 2. Adequacy index of regression model relationship between measured fitness factors and the total score of FMS test of subjects

\begin{tabular}{ccccccc}
\hline Index Model & $\mathbf{r}$ & $\mathbf{r}^{2}$ & Adjusted Coefficient of Determination & Watson Camera & $\mathbf{F}$ & Sig. \\
\hline FMS total test score & 0.095 & 0.01 & 0.01 & 1.976 & 130.270 & 0.856 \\
\hline & & & & & Journal of \\
Sport Biomechanics
\end{tabular}


be examined with fitness factors and the role of gender, and the difference between the subjects. This study concluded no significant relationship between the FMS test and fitness factors' total score. The FMS test considers the quality of movements and basic movements, and the fitness factors take into account sports skills. Each of these tests pursues different goals; therefore, explaining the fitness factors using the FMS test's total score is not possible.

\section{Ethical Considerations}

Compliance with ethical guidelines

All ethical principles are considered in this article. The participants were informed about the purpose of the research and its implementation stages. They were also assured about the confidentiality of their information and were free to leave the study whenever they wished, and if desired, the research results would be available to them.

Funding

This research did not receive any grant from funding agencies in the public, commercial, or non-profit sectors.

Authors' contributions

All authors equally contributed to preparing this article.

Conflicts of interest

The authors declared no conflict of interest.

Acknowledgements

All authors would like to thank the Faculty of Physical Education, University of Guilan. 


\title{
بررسى ارتباط بين آزمون غربالكرى حركتى عملكردى با برخى فاكتورهاى آمادتى جسمانى

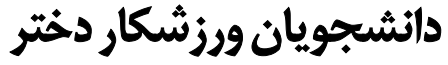

\author{
"مارال انتظامى' هـ، حسن دانشمندى' هـ، على شمسى ماجلان' \\ 1. كروه آسيبشناسى ورزشى و حركات اصلاحى، دانشكده تربيت بدنى و علوم ورزشى، دانشعاه كيلان، رشت، ايران.
}

\begin{abstract}
حكيد
هدف هدف از مطالعه حاضر، بررسى ارتباط بين آزمون غربالكرى حركتى عملكردى با برخى فاكتورهاى آمادكى جسمانى دائشجويان

ورزشكار دختر بود.

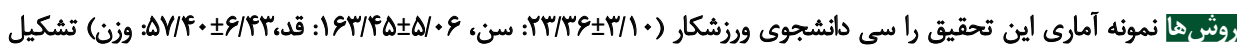

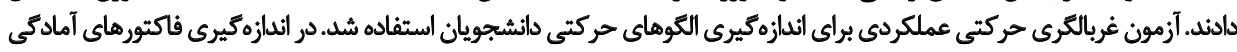

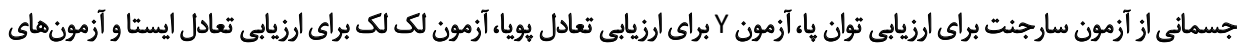

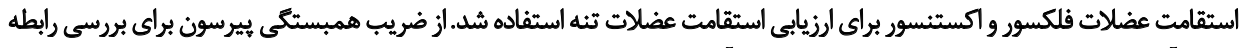

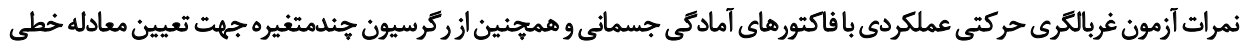

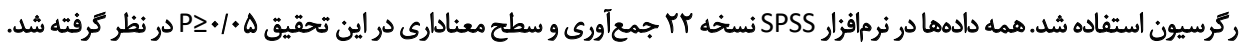

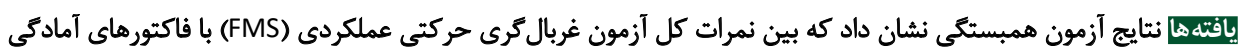

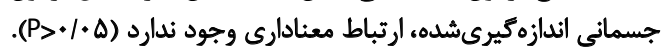

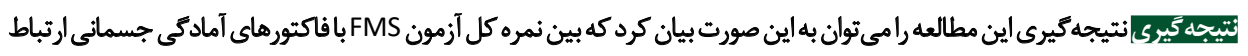

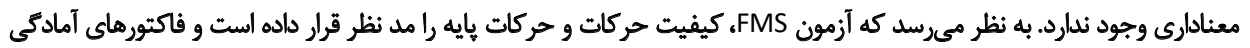

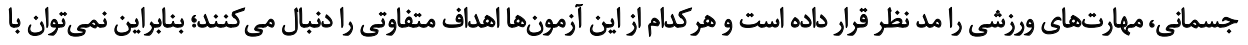

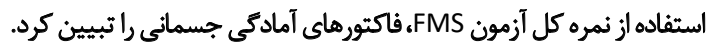

شركت در فعاليتهاى ورزشى روزبهروز گستردهر و به طور

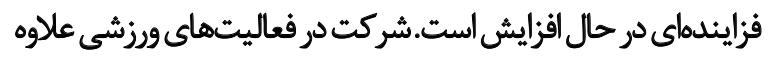

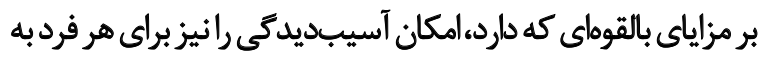

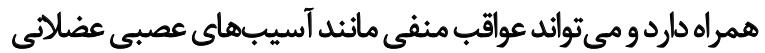

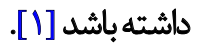

آزمونهاى مختلفى براى ارزيابى كيفيت الكوهاى حركتى تركي

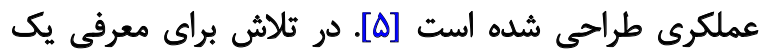

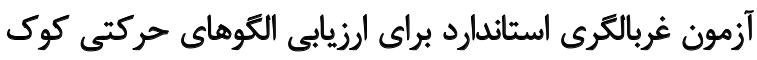

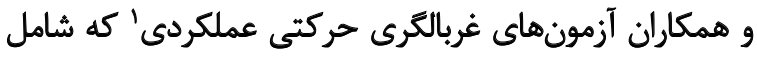

1. Functional Movement Screen (FMS)

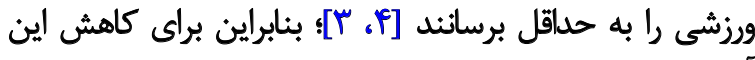

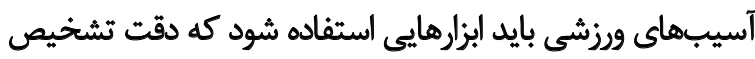
بالايى در ميزان مستعد بونن به آسيب دارند.

\section{-.}

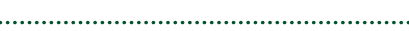

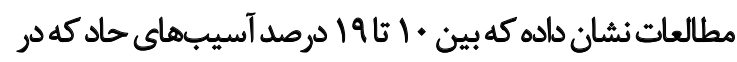

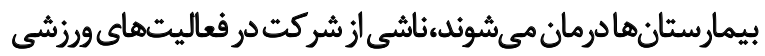

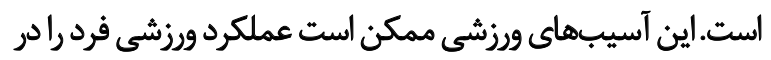

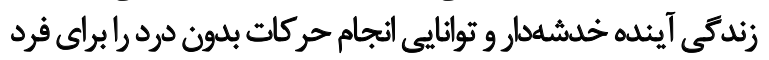

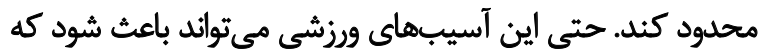

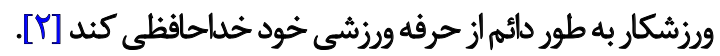


حالى كه در مطالعه ديكر، ارتباط معنادارى بين آزمون FMS ماديا

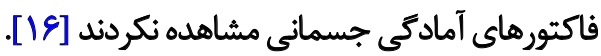
علت متفاوت بودن ارتباط بين نمرات آزمون FMS بافاكتورهاي

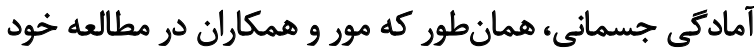

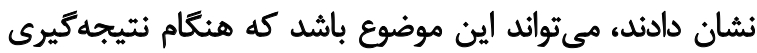

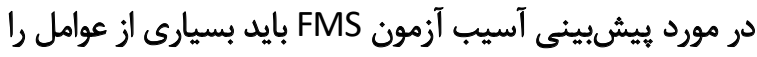
در نظر ترفت و با جزئيات و احتياط بيشترى در مورد مبرد مطالعات

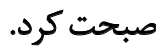

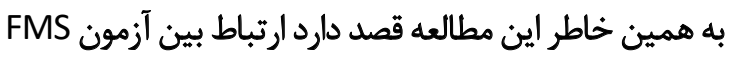

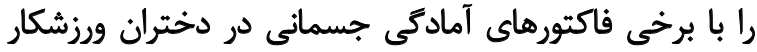

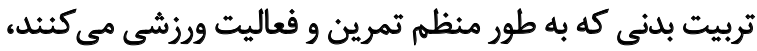

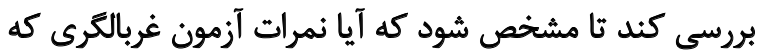

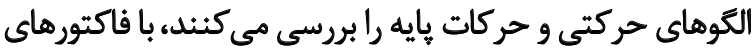

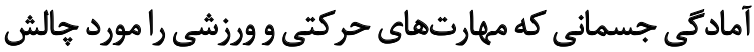

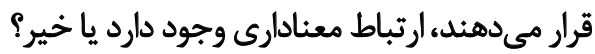

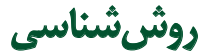

أزمودنىها

يروهش حاضر از نوع همبستكى است. جامعه آمارى تحقيق

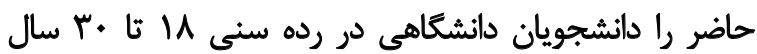

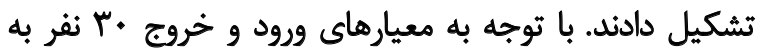

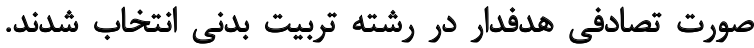

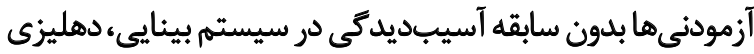

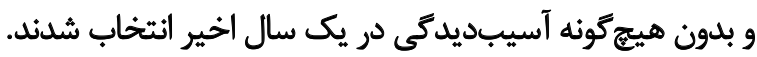
عدم داشتن بيمارى نورولوريكى و ناهنجارى وضعى نيز بر بر روند تحقيق اثركذار بود.

تمام آزمودنى ها موافقت خود را براى مشاركت در اين تحقيق

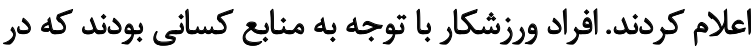

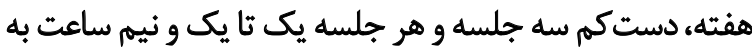

$$
\text { فعاليت ورزشى بيردازند [IV] }
$$

$$
\text { روش اجرا }
$$

تحقيق حاضر در آزمايشكاه دانشكده صورت كرفت و تمام

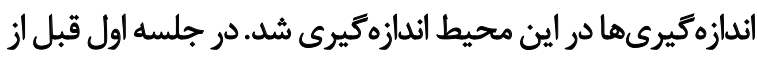

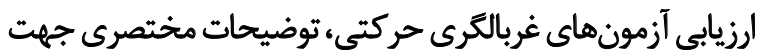

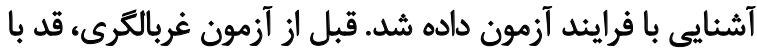

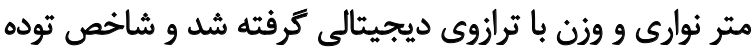

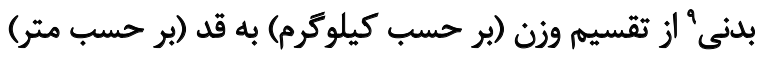

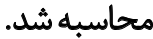

در طول فرايند آزمون غربالكرى حركتى، آزمودنىها بايد با
هفت آزمون ساده (ديب اسكات '، كام برداشتن از روى مانع بَ، لانج

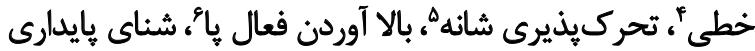

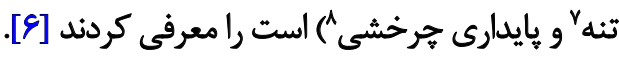

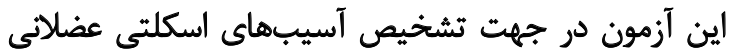

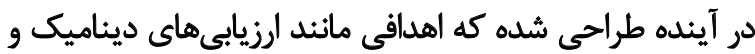

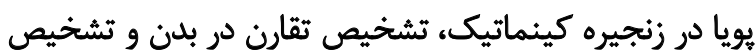

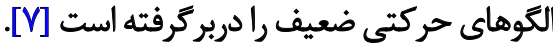
آزمونهاى غربالكرى حركتى عملكردى يك روش سريع،

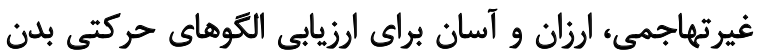

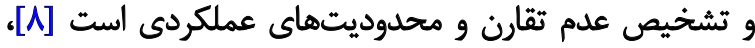

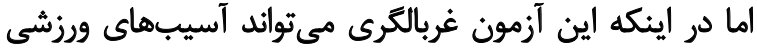

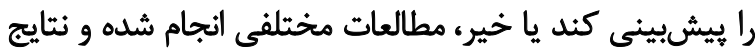
متناقضى هم كزارش كردهاند.

برخى مطالعات نشان دادهاند كه FMS توانايى بيشيشينى و

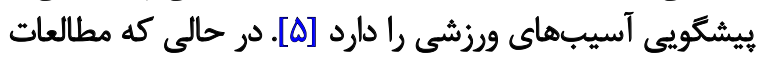

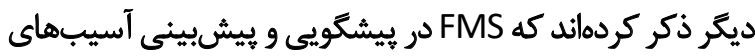

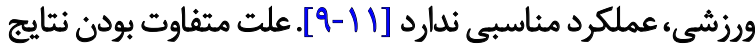

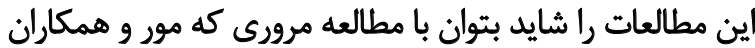

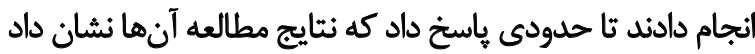

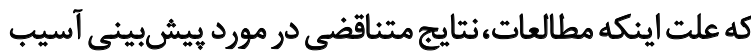

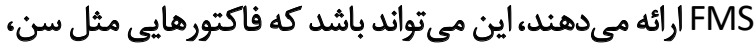

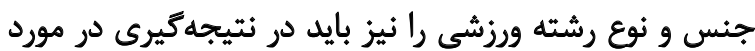

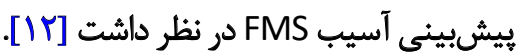

Fلاوه بر اين، بايد مطالعات مختلفى با بررسى ارتباط

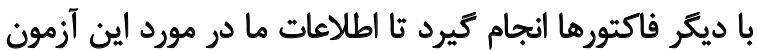

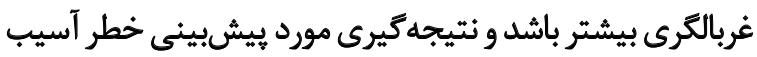

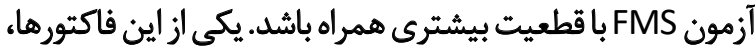

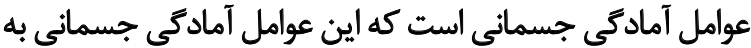
عنوان ريسك فاكتورهاى بيرونى آسيب در مطالعات تأييد شده آمادي

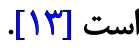

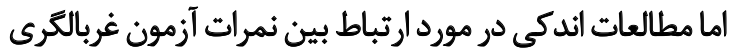

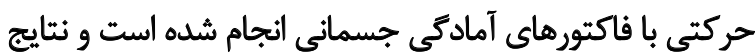

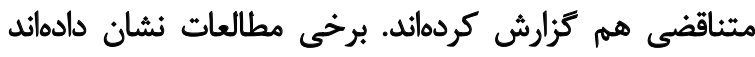

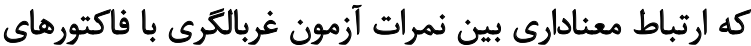

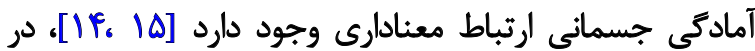

\section{Deep squat}

3. Hurdle step

4. In-line iunge

5. Shoulder mobility

6. Active straight-leg-raise

7. Trunk-stability push-up

8. Rotary stability 
با زمين باشد. براى اجراى آزمون كام از روى مانع آزمودنى، در

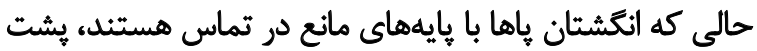

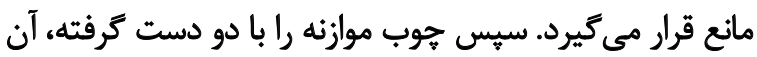

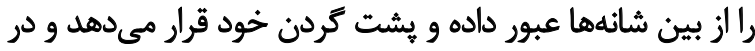

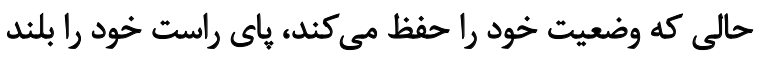

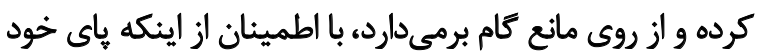

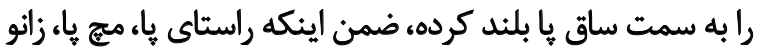
و لكن را بدون بر هم خوردن تعادل حفظ مي كن كند.

سيس كف زمين رابا باشنه لمس كرده و بدون مكث بلافين إصاصله

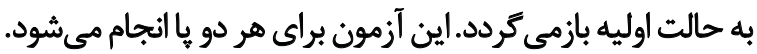

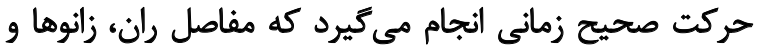

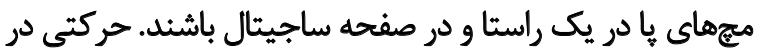
ناحيه كمر اتفاق نيفتد. ميله و مانع با هم موازى باشند.

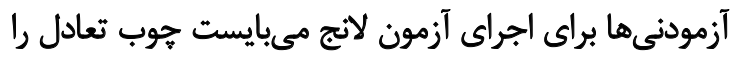

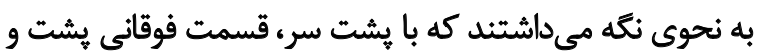

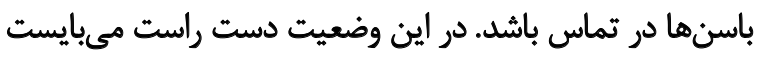

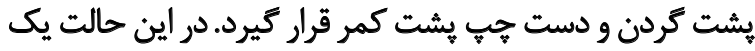

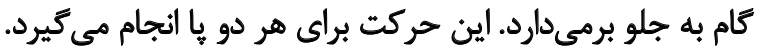

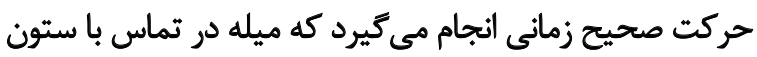

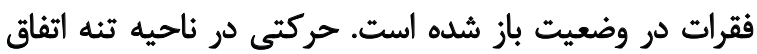

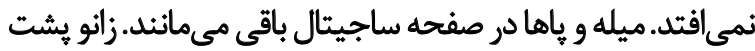
جاشنه ياى جلويى را المس مي كند.

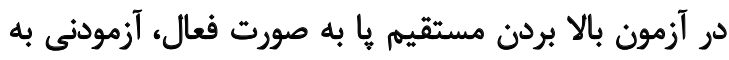

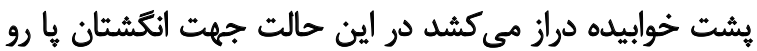

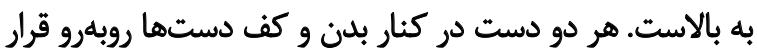

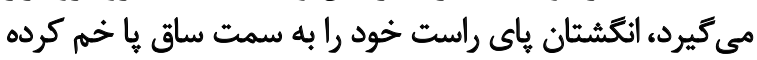

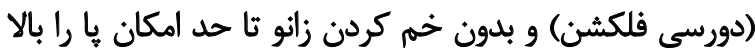

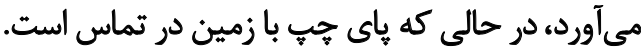

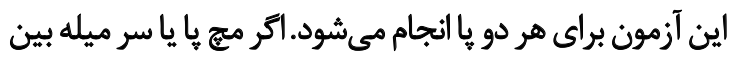

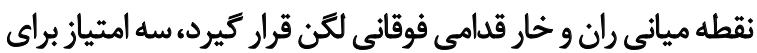

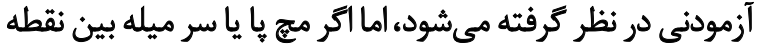

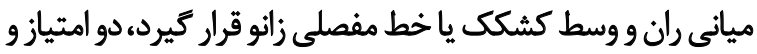

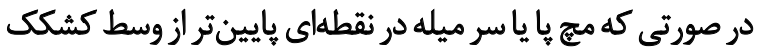

يا خط مفصلى زانو قرار كيرد، يك امتياز به فرد داده مي بـوني

براي انجام آزمون دامنه حركتى شانه فرد، در حالى كه باها

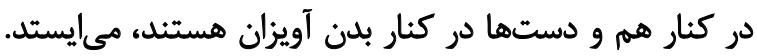

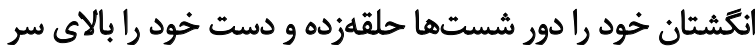

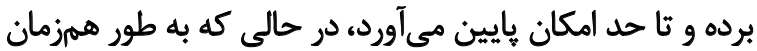

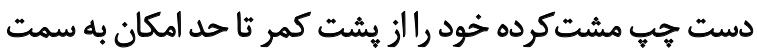

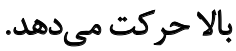

در اين آزمون اكر مشتها در فاصله •r سانتىمترى و ويا

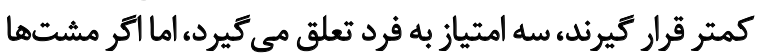

همان وضعيت عادى و كفش خود آزمونهاى غربالكرى را انجام

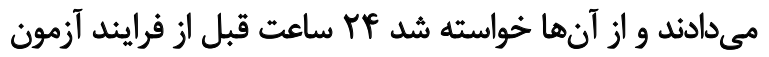

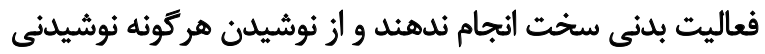

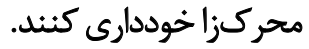

در اين تحقيق براى غربالكرى عملكردى از آزمونهاى FMS (ICC=•/A9)

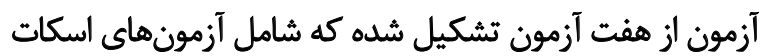

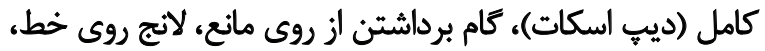

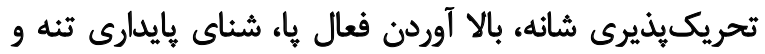

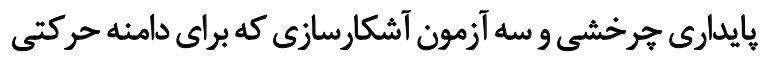
شانه، شناى پايدارى تنه و پايدارى خرخشى است.

توضيحات لازم و دستورالعملهاي كفتارى مربوط بها اجراي هر إن

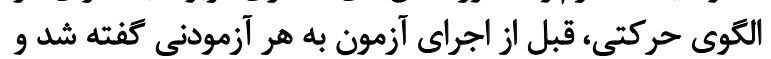

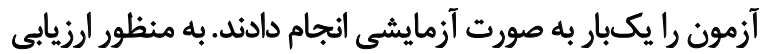

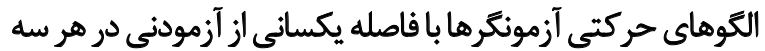

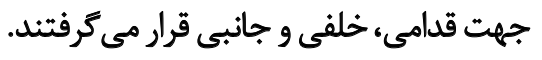

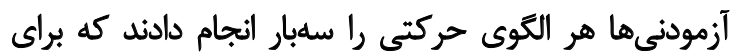

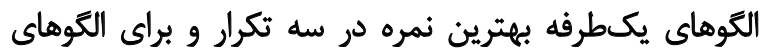

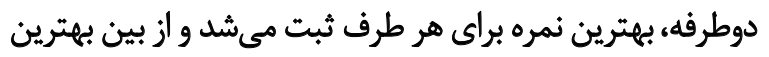

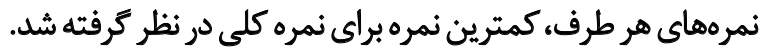
در اين آزمون هر فرد مي تواند نمرات بين صفر تا سه را براى

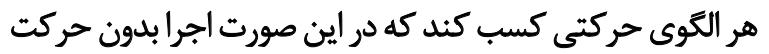

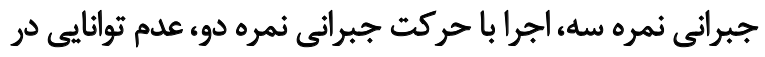

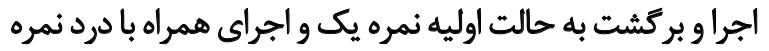
صفر دريافت مى كنيند.

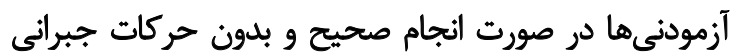

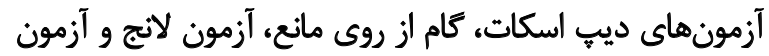

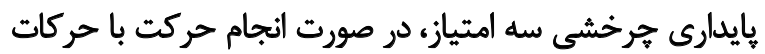

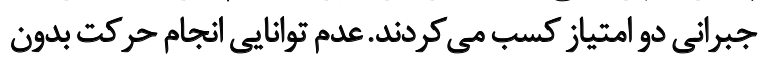

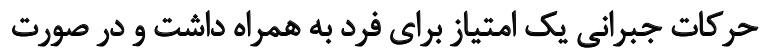

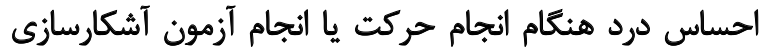

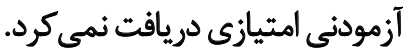

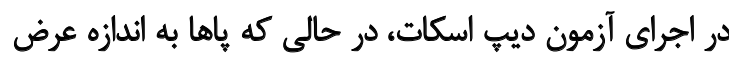

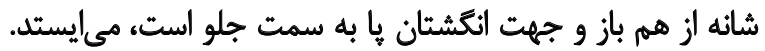

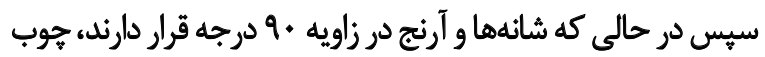

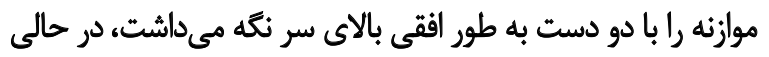

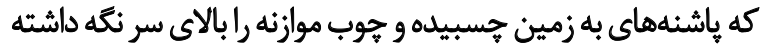

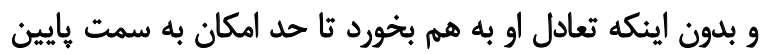

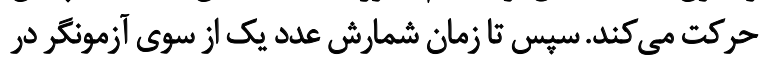

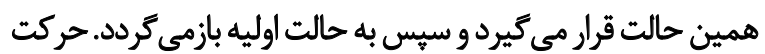

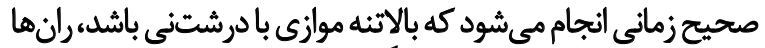

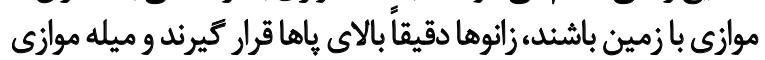




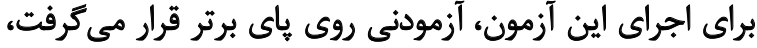

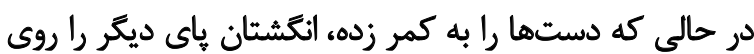

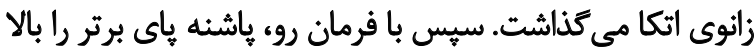

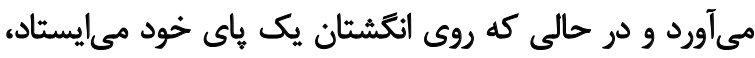

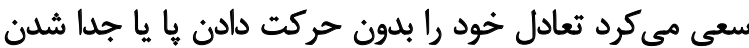
دستها از كمر حفظ كند.

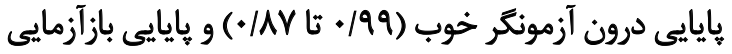

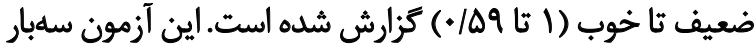

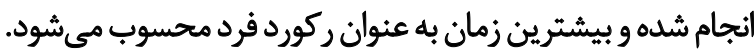

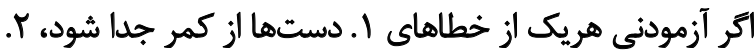

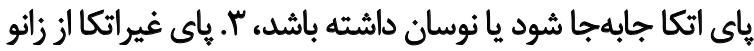

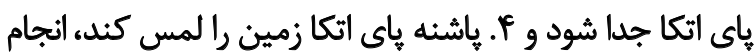

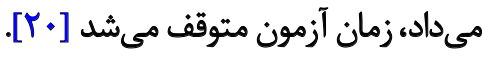

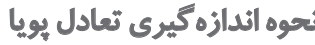

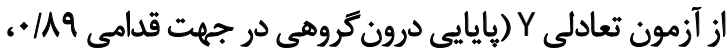

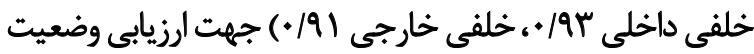

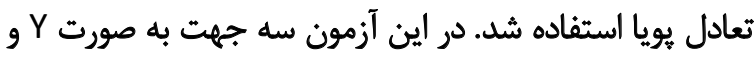

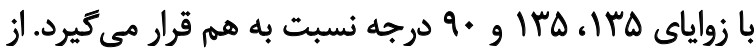

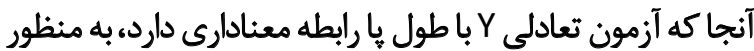

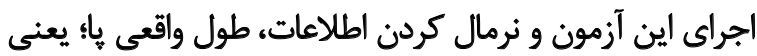

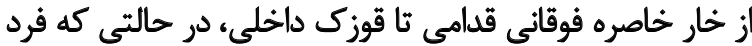

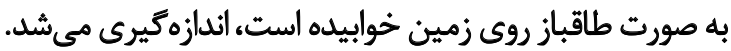
يس إز توضيحات لازم در خصوص نحوه اجراى آزمون توسط

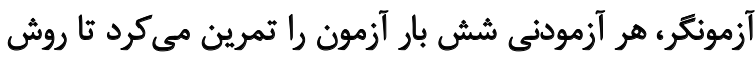

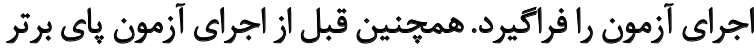

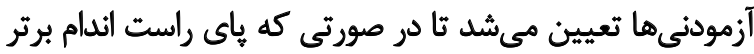

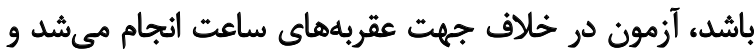

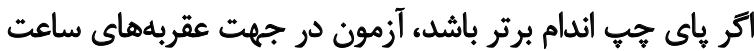

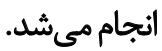

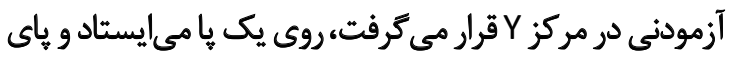

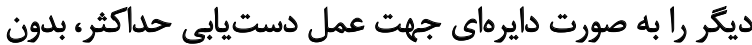

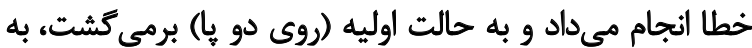

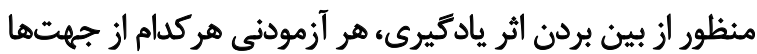
را شش بار با فاصله ها ثانيه استراحت، تمرين مى كردي. بعد از هـ دقيقه استراحت، هر آزمودنى هر يك از جهتها رائرا

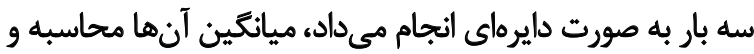

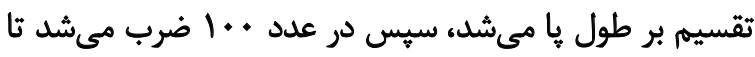

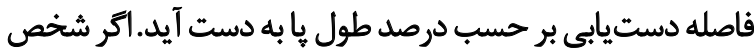

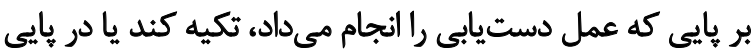

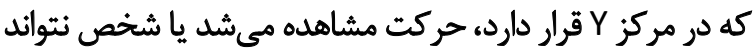

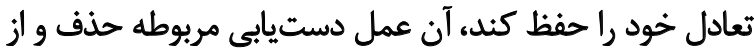

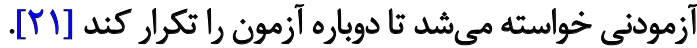

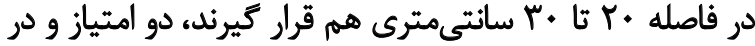

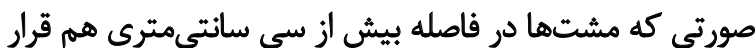
كيرند، يك امتياز به فرد داده مي فئهود.

براى اجراى آزمون شناى بايدارى تنه، آزمودنى در موقعيت

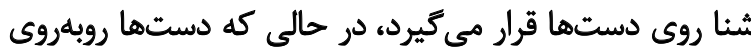

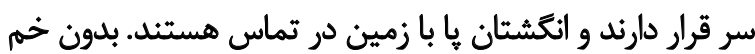

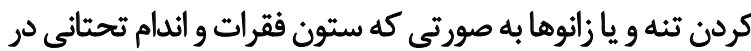

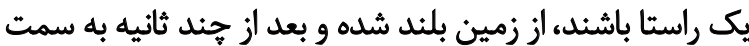

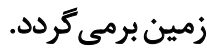

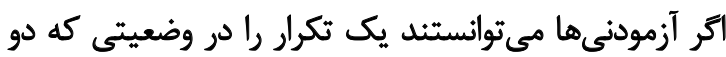

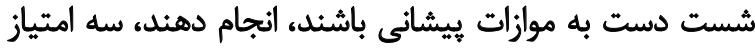

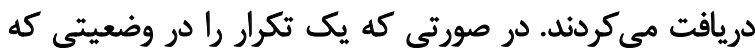

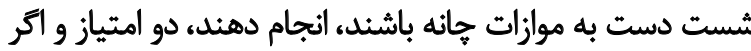
ستون فقرات را در راستاى اندام تحتانى قرار ندهند، يك انداند امتياز

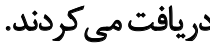

براي اجراى آزمون بايدارى جرخشى، آزمودنى روى تخته تعادل

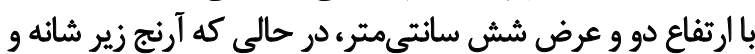

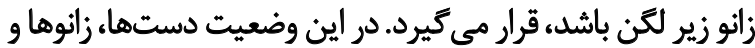

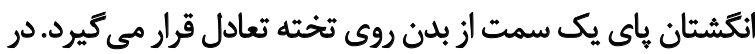

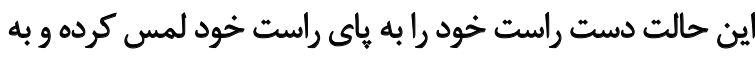

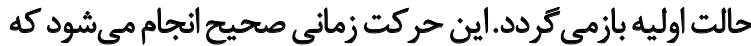

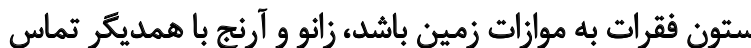
ييدا كنئد و هيجّونه تماسى باز زمين نداشته باشد.

$$
\begin{aligned}
& \text { روش اندازهتيرى فاكتور هاى آمادتّى جسمانى } \\
& \text { نحوه اندازٔهيرى ثوان انفجارى با }
\end{aligned}
$$

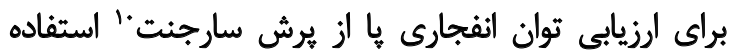

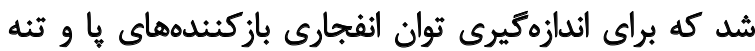

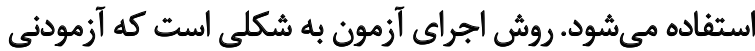

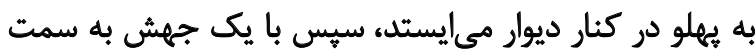

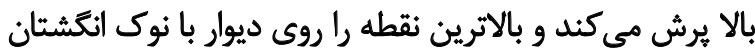

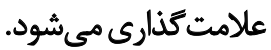

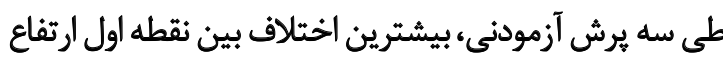

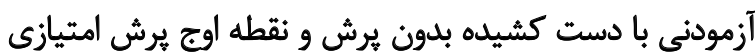

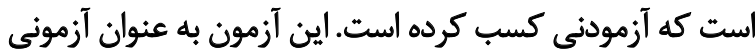

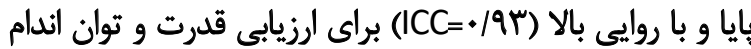

تحتانى كزارش شده است [19].

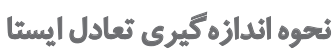
براى اندازهيرى تعادل ايستا از آزمون لكلك" استفاده شد.

10. Jump Sargent

11. Stork Test 
اطلاعات مربوط به ويزٔكىهاى فردى آزمودنىها در جدول

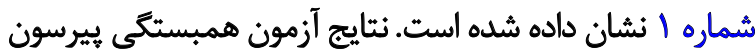

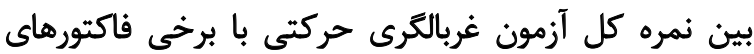

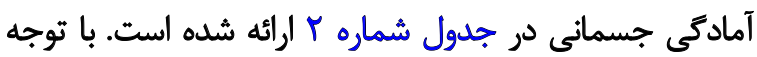

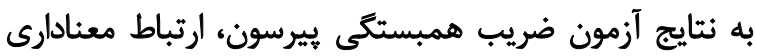

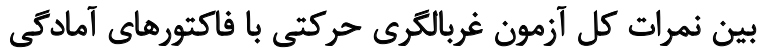
جسمانى اندازهكيرى شده وجود ندارد.

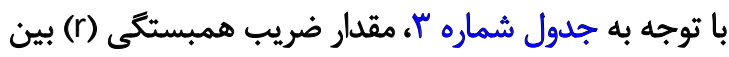

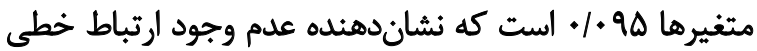

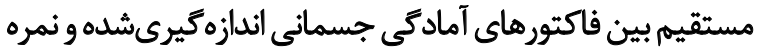

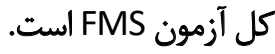

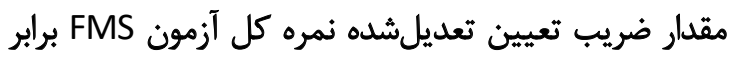

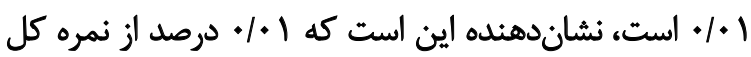

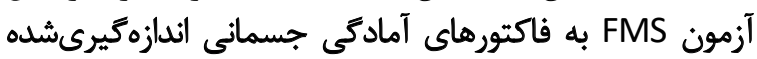

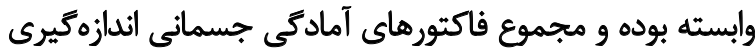

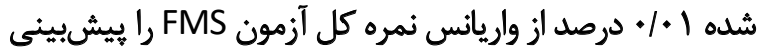

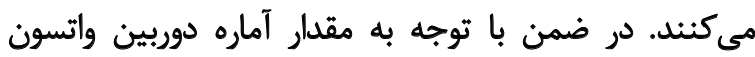
(1/9V9) فرض استقلال خطاها تأييد مىشود.

با توجه به عدم معنادارى مقدار آزمون F در سطح خطاى

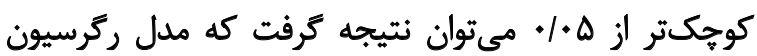

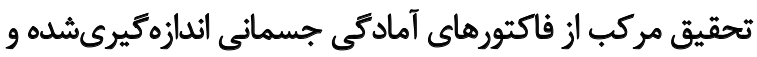

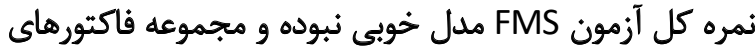

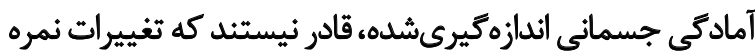

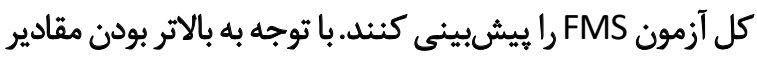
دolerance در يروهش حاضر تأييد مي حالات المد.

بحث

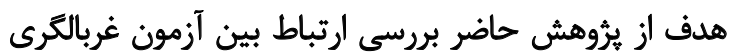

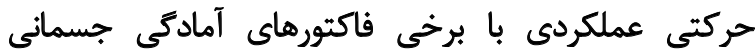

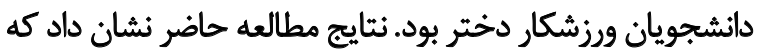

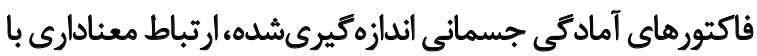

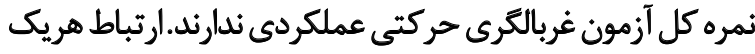

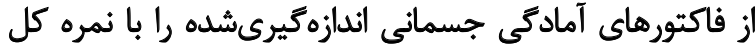
آزمون FMS مورد بحث قرار مي جمهيم.

نتايج حاصل از اين تحقيق عدم ارتباط بين نمره كل آزمون

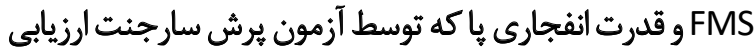

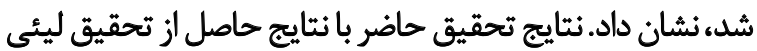

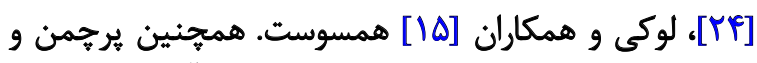
مك بريد در مطالعه خود مشاهده كردند كه بين آزمون غربالكرى همي
براى ارزيابى استقامت عضلات فلكسور تنه از آزمون ارزيابى إيى إئات

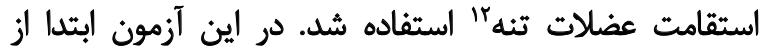

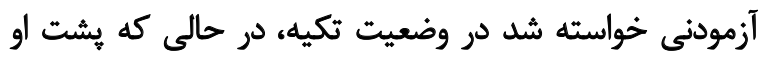

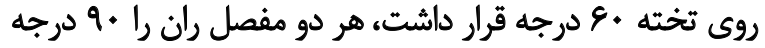

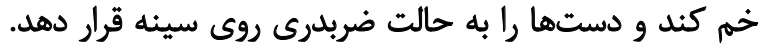

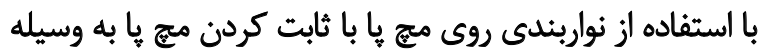

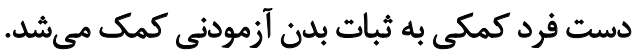
تبراى شروع آزمون، در حالى كه آزمودنى در وضعيت تكيه به تهن

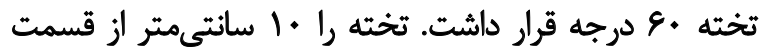

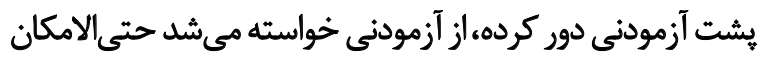

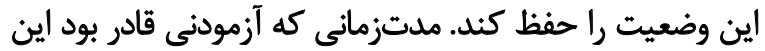

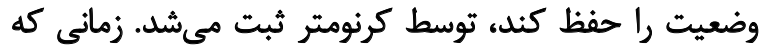

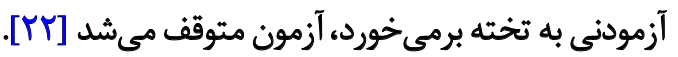

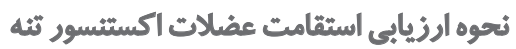

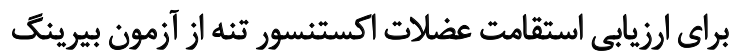

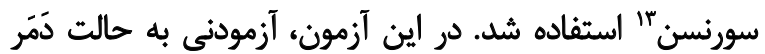

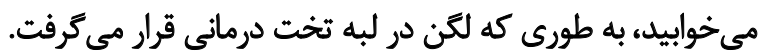

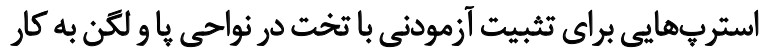

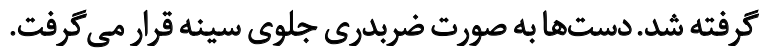
سيس ازفرد خواسته ميشد تنه را موازى سطح زمين نكانه دارد.

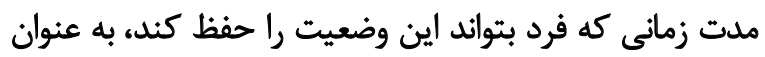

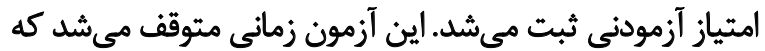

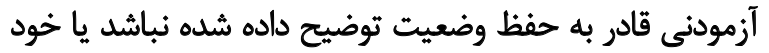

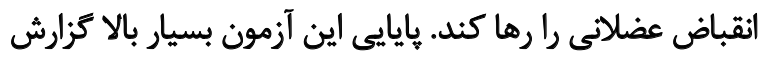

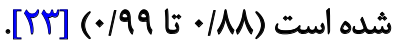
ورشي ثجزيه و تنحليل دادهما در اين تحقيق از آمار توصيفى براى توصيف دادههاى هر تروه

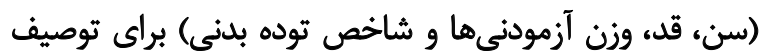

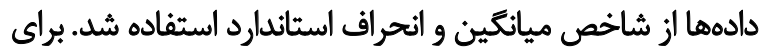

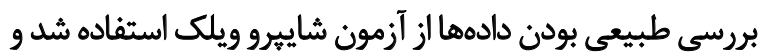

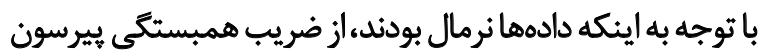

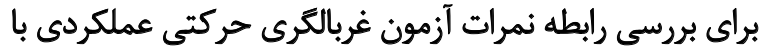

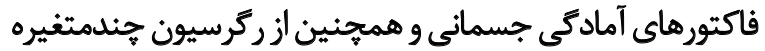

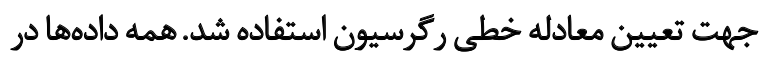

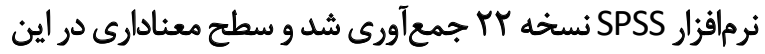

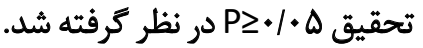

12. Trunk Flexor Endurance Test

13. Biering Sorenson 
جدول ا. ميانكين و انحراف استاندارد مشخصات فردى آزمودئىها

\begin{tabular}{|c|c|}
\hline ميانكين =انحراف معيار & 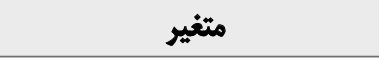 \\
\hline$r r / r E \pm T / l$. & سن (سال) \\
\hline $\mid q T / q \pm \Delta / .9$ & قد (سانتىمتر) \\
\hline$\Delta V / F \cdot \pm S / \mathscr{T H}$ & وزن (كيلوكرم) \\
\hline$r / / r A \pm T / T Y$ & شاخص توده بدن (كيلوكرم بر مترمربع) \\
\hline
\end{tabular}

دارد. نتايج مطالعه حاضر با نتايج مطالعه اشدون و همكاران،

ناهمسوست [Y [ب].

يكى از علتهاى عدم همسو بودن نتايج همين مى تواند باشد

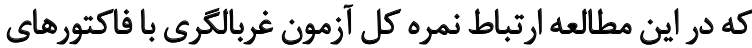

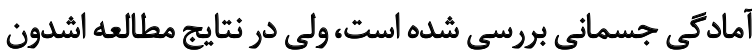

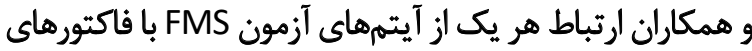

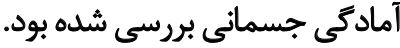

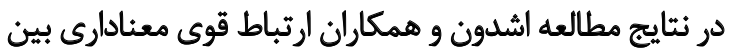

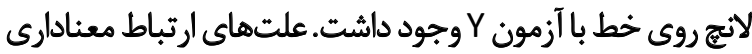

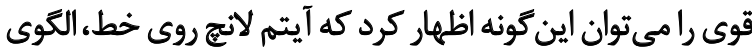

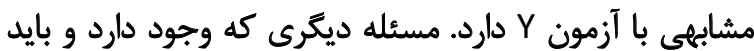

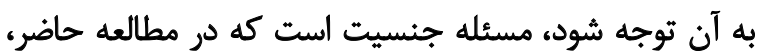

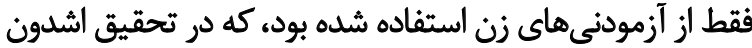

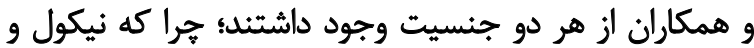

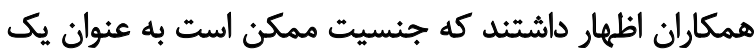

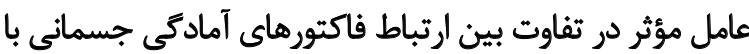

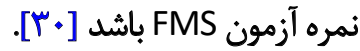

حركتى و برخى فاكتورهاى آمادگى جسمانى همجيون يرش سارجنت ارتباط معنادارى وجود نداردي آدر اين تحقيق بيان شد كه FMS يك ابزار مناسب براى

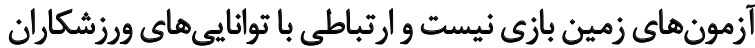

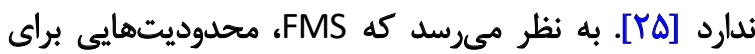

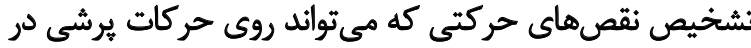

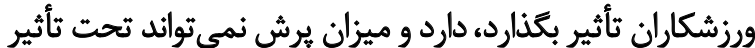

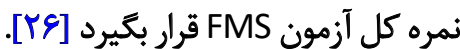
به نظر مىرسد سرعت و توان يا تحت تأثير عواملى، از جمله

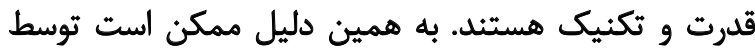

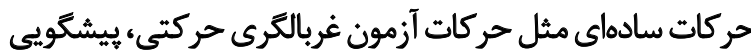

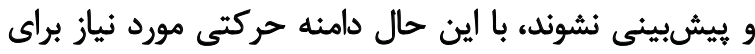

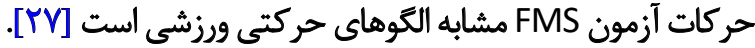
در تحقيق حاضر بين نمره كل آزمون FMS و تعادئ تعادل ايستا

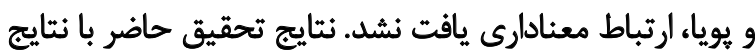

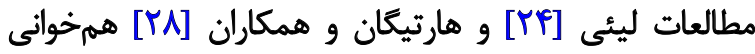

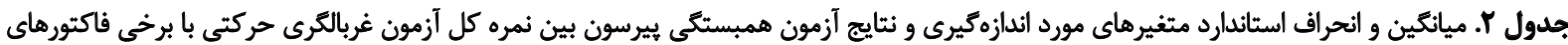

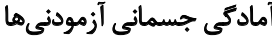

\begin{tabular}{|c|c|c|c|c|}
\hline \multicolumn{3}{|c|}{ نمره كل آزمون غربال كرى حركتى عملكردى } & \multirow{2}{*}{ ميانكين =|نحراف|ستاندارد } & \multirow{2}{*}{ فاكتور هاى آمادكى جسمانى } \\
\hline Sig. & $r^{r}$ & $\mathbf{r}$ & & \\
\hline - Nar & +1 & $.1 \cdot 8$ & $M \varphi / \varphi+ \pm \Delta / \Delta \mu$ & توان ائفجارى يا (ساتتىمتر) \\
\hline ./QYA & $1 \cdots$ & $.1 \cdot 18$ & $\mid r / \varepsilon A \pm \Delta / \gamma$ & تعادل ايستا (ثانيه) \\
\hline Mqr & $\%$ & $.1 .8 r$ & $A r / Q \pm N / F F$ & تعادل يويا (درصد طول با) \\
\hline - IASA & $\%$ & $.1 \cdot 19$ & $1.9 / 19 \pm \Delta N / q q$ & استقامت عضلات فلكسور تنه (ثانيه) \\
\hline.$/ 2 \mathrm{ra}$ & $.1+1$ & . n & 1.V/११+YNES & استقامت عضلات اكستنسور ثنه (ثانيه) \\
\hline
\end{tabular}

جدول لا. شاخص كفايت مدل ركرسيونى رابطه فاكتورهاي آمادگى جسمانى ائدازهيرى شده با نمره كل آزمون غربالكرى حركتى عملكردى آزمودنىها

\begin{tabular}{|c|c|c|c|c|c|c|}
\hline Sig. & $\mathbf{F}$ & دوربين واتسون & ضريب تعيين تعديلشده & $r^{r}$ & $\mathbf{r}$ & شاخص مدل \\
\hline - /ASE & 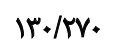 & V/ave &.$/ 1$ & $.1+1$ & .1 .90 & FMS نمره كل آزمون \\
\hline
\end{tabular}


جسمانى و مهارتهاي ورزشى تأكيد داشته است. هركدام از اين

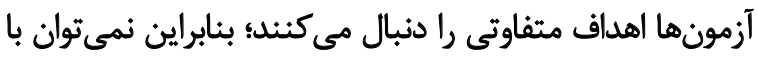

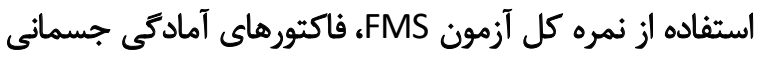

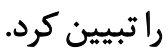

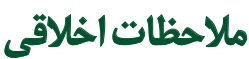

\section{يبيروى از اصول اخلاق يثوهش}

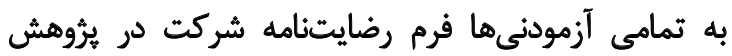

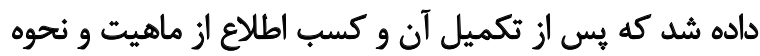

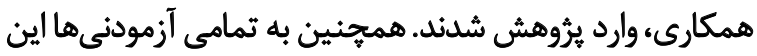

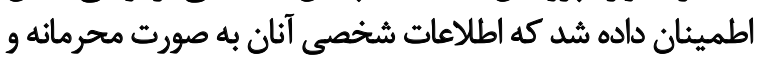

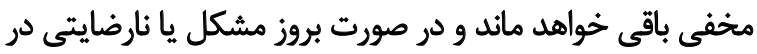

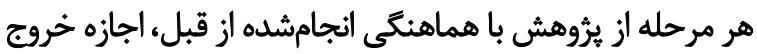

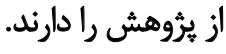

$$
\text { حامى مالى }
$$

اين تحقيق هيج كُونه كمك مالى از سازمان هاي تأمين مالى در بخش هاى عمومى ، تجارى يا غير انتفاعى دريافت كمئ نكرد.

$$
\text { مشاركت نويسندكان }
$$

تمام نويسندكان در طراحى، اجرا و نكارش همه بخشهاي يرؤشش حاضر مشاركت داشتهاند.

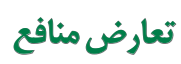

اين مقاله هيجّونه تعارض منافى ندارد.

$$
\text { تشكر و قدردانى }
$$

بدينوسيله از دانشكده تربيت بدنى دانشعاه كيلان و آزمودنىها

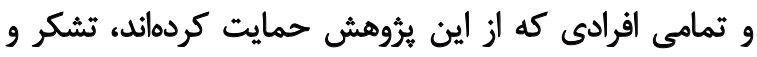

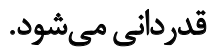

همجنين نتايج مطالعه حاضر نشان داد كه ارتباط معنادارى

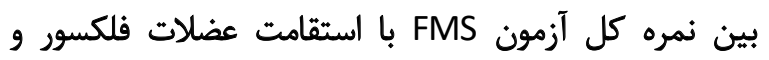

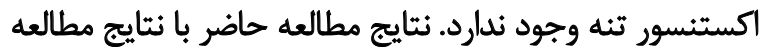

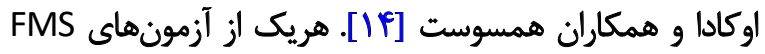

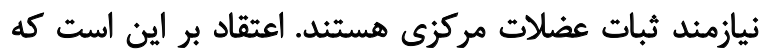

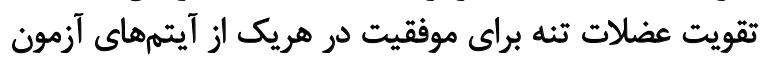
FMS

$$
\text { به نظر مىرسد. }
$$

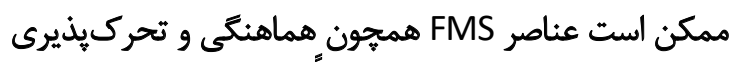

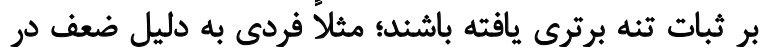

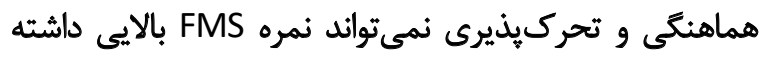

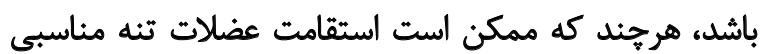
داشته باشد. با اين حال توجيه اين مسئله است دشوار است.

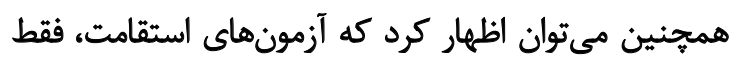

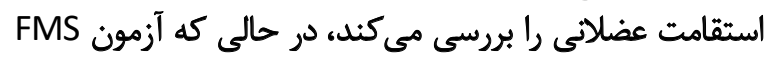

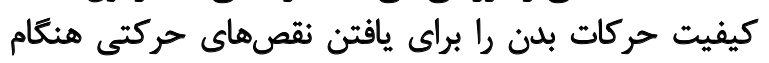

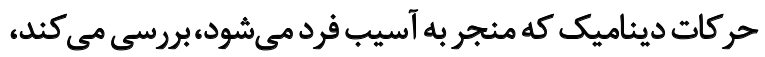

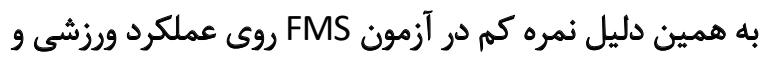

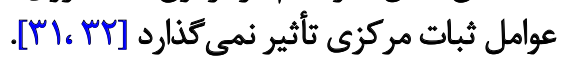
از طرفى نتايج تحقيق حاضر به نتايج تحقيقى كه ميجحل و و

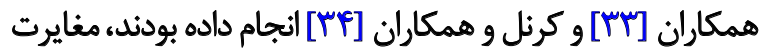

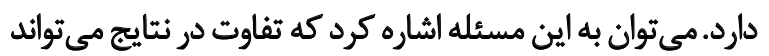

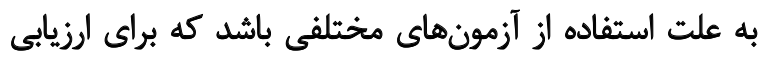

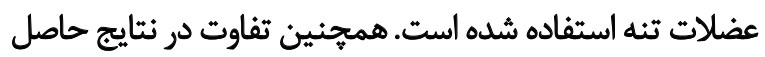

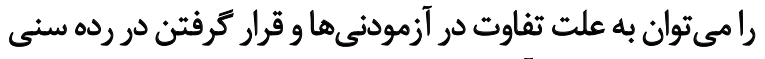
متفاوت و جنسيت آزمودنى ها دانست.

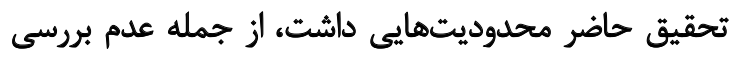

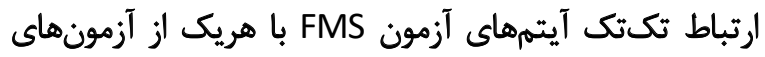

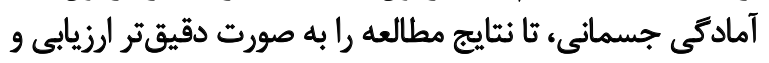

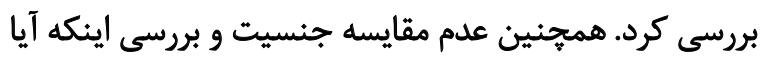

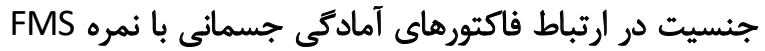
نقش دارد يا خير.

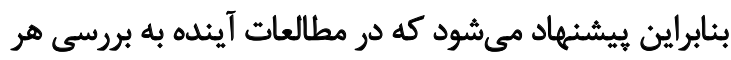

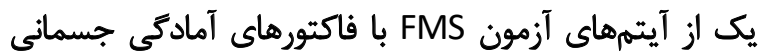

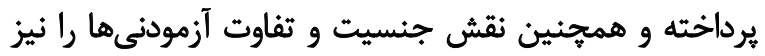

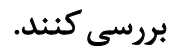

\section{نتيجليَّيرى نهايى}

نتيجه كيرى اين مطالعه را مى توان به اين صورت بيان كرد كه إنها

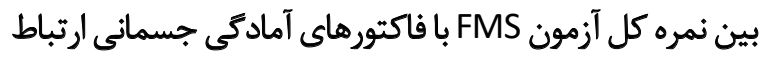

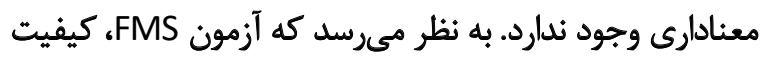
حركات و حركات بايه را مد نظر قرار داده و بر بر فاكتورهاى آمادئى 


\section{References}

[1] Caine DJ, Provance AJ. Pediatric and adolescent injury in adventure and extreme sports. Res Sports Med. 2018; 26(suppl 1):5-19. [DOI:1 0.1080/15438627.2018.1434041] [PMID]

[2] Patel DR, Yamasaki A, Brown K. Epidemiology of sports-related musculoskeletal injuries in young athletes in United States. Transl Pediatr. 2017; 6(3):160-6. [DOI:10.21037/tp.2017.04.08] [PMID] [PMCID]

[3] Bakken A, Targett S, Bere T, Eirale C, Farooq A, Tol JL, et al. The functional movement test $9+$ is a poor screening test for lower extremity injuries in professional male football players: A 2-year prospective cohort study. Br J Sports Med. 2018; 52(16):1047-53. [DOI:10.1136/ bjsports-2016-097307] [PMID]

[4] Walbright PD, Walbright N, Ojha H, Davenport T. Validity of functional screening tests to predict lost-time lower quarter injury in a cohort of female collegiate athletes. Int J Sports Phys Ther. 2017; 12(6):948-59. [DOI:10.26603/ijspt20170948] [PMID] [PMCID]

[5] Bonazza NA, Smuin D, Onks CA, Silvis ML, Dhawan A. Reliability, validity, and injury predictive value of the functional movement screen: A systematic review and meta-analysis. Am J Sports Med. 2017; 45(3):725-32. [DOI:10.1177/0363546516641937] [PMID]

[6] Cook G, Burton L, Hoogenboom B. Pre-participation screening: The use of fundamental movements as an assessment of function - part 1. N Am J Sports Phys Ther: NAJSPT. 2006; 1(2):62-72. [PMID] [PMCID]

[7] Monaco J-T, Schoenfeld BJ. A review of the current literature on the utility of the functional movement screen as a screening tool to identify athletes' risk for injury. Strength Cond J. 2019; 41(5):17-23. [DOI:10.1519/SSC.0000000000000481]

[8] Dyer CS, Callister R, Sanctuary CE, Snodgrass SJ. Functional Movement Screening and injury risk in elite adolescent rugby league players. Int J Sports Sci Coach. 2019; 14(4):498-506. [DOI:10.1177/1747954119853650]

[9] Dossa K, Cashman G, Howitt S, West B, Murray N. Can injury in major junior hockey players be predicted by a pre-season functional movement screen-a prospective cohort study. J Can Chiropr Assoc. 2014; 58(4):421-7. [PMID] [PMCID]

[10] Moran RW, Schneiders AG, Mason J, Sullivan SJ. Do Functional Movement Screen (FMS) composite scores predict subsequent injury? A systematic review with meta-analysis. Br J Sports Med. 2017; 51(23):1661-9. [DOI:10.1136/bjsports-2016-096938] [PMID]

[11] Dorrel BS, Long T, Shaffer S, Myer GD. Evaluation of the functional movement screen as an injury prediction tool among active adult populations: A systematic review and meta-analysis. Sports Health. 2015; 7(6):532-7. [DOI:10.1177/1941738115607445] [PMID] [PMCID]

[12] Moore E, Chalmers S, Milanese S, Fuller JT. Factors influencing the relationship between the functional movement screen and injury risk in sporting populations: A systematic review and meta-analysis. Sports Med. 2019; 49(9):1449-63. [DOI:10.1007/s40279-019-011265] [PMID]

[13] Emery CA. Risk factors for injury in child and adolescent sport: A systematic review of the literature. Clin J Sport Med. 2003; 13(4):256-68. [DOI:10.1097/00042752-200307000-00011] [PMID]

[14] Okada T, Huxel KC, Nesser TW. Relationship between core stability, functional movement, and performance. J Strength Cond Res. 2011; 25(1):252-61. [DOI:10.1519/JSC.0b013e3181b22b3e] [PMID]

[15] Lockie RG, Schultz AB, Callaghan SJ, Jordan CA, Luczo TM, Jeffriess MD. A preliminary investigation into the relationship between functional movement screen scores and athletic physical performance in female team sport athletes. Biol Sport. 2015; 32(1):41-51. [DOI:10.5604/20831862.1127281] [PMID] [PMCID]

[16] Parchmann CJ, McBride JM. Relationship between functional movement screen and athletic performance. J Strength Cond Res. 2011; 25(12):3378-84. [DOI:10.1519/JSC.0b013e318238e916] [PMID]

[17] Rejali M, Mostajeran M. Assessment of physical activity in medical and public health students. J Educ Health Promot. 2013; 2:19. [DOI:10.4103/2277-9531.112690] [PMID] [PMCID]

[18] Cook G, Burton L, Hoogenboom BJ, Voight M. Functional Movement Screening: The use of fundamental movements as an assessment of function - part 2. Int J Sports Phys Ther. 2014; 9(4):549-63. [PMID] [PMCID]

[19] Ayán-Pérez C, Cancela-Carral JM, Lago-Ballesteros J, Martínez-Lemos I. Reliability of sargent jump test in 4-to 5-year-old children. Percept Mot Skills. 2017; 124(1):39-57. [DOI:10.1177/0031512516676174] [PMID]

[20] Sever O. [Comparison of static and dynamic core exercises' effects on Stork balance test in soccer players (Turkish)]. J Hum Sci. 2017; 14(2):1781-91. [DOI:10.14687/jhs.v14i2.4440]

[21] Plisky PJ, Gorman PP, Butler RJ, Kiesel KB, Underwood FB, Elkins B. The reliability of an instrumented device for measuring components of the star excursion balance test. N Am J Sports Phys Ther: NAJSPT. 2009; 4(2):92-9. [PMID] [PMCID]

[22] Juan-Recio C, López-Plaza D, Barbado Murillo D, García-Vaquero MP Vera-García FJ. Reliability assessment and correlation analysis of 3 protocols to measure trunk muscle strength and endurance. J Sports Sci. 2018; 36(4):357-64. [DOI:10.1080/02640414.2017.1307439] [PMID]

[23] Applegate ME, France CR, Russ DW, Leitkam ST, Thomas JS. S $\varnothing$ rensen test performance is driven by different physiological and psychological variables in participants with and without recurrent low back pain. J Electromyogr Kinesiol. 2019; 44:1-7. [DOI:10.1016/j. jelekin.2018.11.006] [PMID]

[24] Zou L. Relationship between Functional Movement Screening and skill-related fitness in college students. Int J Sports Sci. 2016; 6(1):11-8. https://www.researchgate.net/publication/301649064_Relationship_between_Functional_Movement_Screening_and_Skill-Related_Fitness_in_College_Students

[25] Parchmann CJ, McBride JM. Relationship between Functional Movement Screen and athletic performance. J Strength Cond Res. 2011; 25(12):3378-84. [DOI:10.1519/JSC.0b013e318238e916] [PMID]

[26] Butler RJ, Plisky PJ, Southers C, Scoma C, Kiesel KB. Biomechanical analysis of the different classifications of the Functional Movement Screen deep squat test. Sports Biomech. 2010; 9(4):270-9. [DOI:10.1 080/14763141.2010.539623] [PMID]

[27] Li Y, Wang X, Chen X, Dai B. Exploratory factor analysis of the Functional Movement Screen in elite athletes. J Sports Sci. 2015; 33(11):1166-72. [DOI:10.1080/02640414.2014.986505] [PMID]

[28] Hartigan EH, Lawrence M, Bisson BM, Torgerson E, Knight RC. Relationship of the functional movement screen in-line lunge to power, speed, and balance measures. Sports Health. 2014; 6(3):197-202 [DOI:10.1177/1941738114522412] [PMID] [PMCID]

[29] Ashdown SC. Relationship between stabilization, balance, athletic performance and functional movement [MSc. thesis]. 2013; Vereinigte Staaten: Brigham Young University. https://scholarsarchive.byu. edu/cgi/viewcontent.cgi ?article $=4694 \&$ context=etd 
[30] Chimera NJ, Smith CA, Warren M. Injury history, sex, and performance on the functional movement screen and $Y$ balance test. J Athl Train. 2015; 50(5):475-85. [DOI:10.4085/1062-6050-49.6.02] [PMID] [PMCID]

[31] Kazman JB, Galecki JM, Lisman P, Deuster PA, O'Connor FG. Factor structure of the functional movement screen in marine officer candidates. J Strength Cond Res. 2014; 28(3):672-8. [DOI:10.1519/ JSC.0b013e3182a6dd83] [PMID]

[32] Bagherian S, Ghasempoor K, Rahnama N, Wikstrom EA. The effect of core stability training on functional movement patterns in college athletes. J Sport Rehabil. 2019; 28(5):444-9. [DOI:10.1123/jsr.2017-0107] [PMID]

[33] Mitchell UH, Johnson AW, Adamson B. Relationship between functional movement screen scores, core strength, posture, and body mass index in school children in Moldova. J Strength Cond Res. 2015; 29(5):1172-9. [DOI:10.1519/JSC.0000000000000722] [PMID]

[34] Cornell DJ, Gnacinski SL, Zamzow A, Mims J, Ebersole KT. Measures of health, fitness, and functional movement among firefighter recruits. Int J Occup Saf Ergon. 2017; 23(2):198-204. [DOI:10.1080/10803548.2016. 1187001] [PMID] 